\title{
The Automatic Undistortion Strength Estimation for Any Describable Optical Distortion
}

\author{
Natalija Chmelarova, Pavel Chmelar, Lubos Rejfek \\ Department of Electrical Engineering \\ Faculty of Electrical Engineering and Informatics, University of Pardubice \\ Pardubice, Czech Republic \\ natalija.chmelarova@student.upce.cz
}

\begin{abstract}
A presence of an optical distortion does undesired influence on the geometrical image quality. The main reason of its occurring is using a lens with low or variable focal length or simple camera optics. When an image captured by a camera is affected by undesired distortion and it is used for the distance measurement, it can cause significant measurement errors. The aim of this paper is to introduce a method for finding an optimal undistortion parameter for any optical distortion, which can be described mathematically. The proposed method uses the Hough transformation accumulator and at each iteration step the quality of straight lines is evaluated as a gradient from local maximum. The algorithm is verified and assessed on several images. The developed approach can be used to find the global maximum gradient in general, even when a local maximum changes its size over iterations.
\end{abstract}

Keywords-optical distortion, distance measurement, Hough transform, gradient method

\section{INTRODUCTION}

The wide angle camera lenses are often used in modern embedded systems, especially when the camera needs to cover wide viewing angle like robotic systems or 3D space scanning application. These lenses satisfy system's requirements but an optical distortion can occur. Its presence causes undesired influence on processed images, especially when segmented object's pixels are used for the physical position determination or directly for the distance estimation. The optical distortion has to be eliminated before a camera frame is further processed.

There are many optical distortions, see [1] or [2], with different reasons of their occurring. The main reasons can be using a simple camera optics or using a low focal length. In the normal case the light rays' incidence angle is equal to the exit angle. When this condition is not fulfilled and lens assembly is not used, the optical distortion will occur.

The recent research [3] deals with the fisheye barrel distortion effect. The correction method uses the benefit of the straight slope constraint. For the undistortion parameter estimation it is needed a single image with grid lines. Authors estimated the correction precision better than half of a pixel. Similar to this describes also paper [4]. The next research presented in [5] uses multiple nearest neighbor-based interpolation for the correction parameter estimation. This approach shows the efficiency also on the strong distortion effect. The interpolation also filtrates the output image. The research in [6] presents the correction algorithm based on collinearity condition and k-nearest neighbor search. The authors focus especially on wide range field of view (FOV). The proposed approach offers better results than conventional methods especially when wide angle range is used. Another correction method presented in [7] is based on the plumb-line approach which uses the Fitzgibbon's division model, robust estimation of circular arcs. Several RANSAC methods for

The research was supported by the internal SGS project University of Pardubice No. SGS 2019021. fitting circle parameters are compared for their robustness and the processing speed in this research. The research in [8] deals with a following correction method, which uses the nine points correspondence presenting the nine bilinear equation system. The algorithm is able to deal with many radial distortions and only two images are necessary for correction parameter estimation.

For the whole processing speed-up and real time applications modern GPUs, DSP systems, and devices with FPGA are used, where image data are thoroughly processed in parallel. For example, authors in [9] use the OpenGL library to accelerate the correction process. The results show over 190 times speed-up against the same implementation on the CPU and at least 50 times in the worst case with the processing time about $1 \mathrm{~ms}$. The similar research in [10] shows implementation on the DSP system where one frame is processed in $50 \mathrm{~ms}$. The best speed result is achieved by a system with the FPGA presented in [11]. The correction process took only $13 \mathrm{~ns}$. When high resolution frames are used the authors recommend to use the fast external memory DDR2 or DDR3. All implementations in [9], [10], and [11] are compared in images with similar resolutions.

Problems with optical distortions occur not only in normal cameras. One of the most recent research in [12] faces to the distortion also in microscopy systems, where the determination of the precise pixel position is important. The achieved correction and advanced image processing ensures the capability to simultaneously resolve multiple biological components using a compact spectral endoscopy system.

In one of our recent papers [13] we are dealing with simple algorithm for barrel distortion correction. The used camera lens has wide angle range especially for increasing the vertical FOV, which increases the number of measurement points along the $Z$ axis. The proposed method includes also the super-sampling filter for the quality enhancement in lower resolution than HD. The whole process speed-up is achieved by the undistortion matrix, used as lookup table. Our paper lacks of the optimal undistortion strength parameter estimation. Similar to research in [4], [10], and [11] our implementation also does not require priori knowledge about the camera and lens parameters. Mentioned papers describe the undistortion strength or the correction factor. But witch parameter is the best for the given lens and camera configuration? The aim of this paper is to introduce a method for finding the optimal correction parameter by the Hough transformation accumulator and the gradient method.

The rest of the paper is organized as follows. Section II describes the details of the proposed algorithm and how to appropriately use its individual tools to achieve the best result. Section III includes comparison and evaluation of achieved results with recommendations for the algorithm utilization. The paper ends with a conclusion. 


\section{OPtimAl StREngth PARAMETER Estimation}

The size of the correction parameter, $C P$, is possible to estimate experimentally. An image, where the undistortion quality is assessed, has to be captured on a scene with the presence of straight lines, like windows, doors, tables, and room's edges are, similar to Fig. 1 (a). From comparison of several undistortion algorithm's outputs we can get the best parameter.

The experimental searching is time consuming and can lead to imprecise estimation. Thus in this paper we propose the new algorithm for the best correction of strength parameter estimation. The distortion effect causes the straight lines curvature. This effect is increasing in bigger pixel distance from the centre of an image. Therefore, the algorithm is based on straight line evaluation from the Hough transformation accumulator. The Hough accumulator summarise lines' presence in an input image for the defined angle range. The accumulator's point intensity expressing the number of line points. A line can be also discontinuous. The idea behind the best parameter estimation is the mathematical evaluation of the resultant image quality instead the visual inspection. It follows that we have to process the input image with the defined $C P$ range and then mathematically evaluate the resultant images.

The bigger point's intensity means more line points at angle determined by the accumulator's column. The correctly undistorted line will have the biggest points' amount and vice versa. The proposed algorithm is based on this feature. But only find of all local maxima is not sufficient, because the number of a line points are changing with the correction parameter size. The main idea behind the algorithm is described below. The first algorithm step is the image edge detection.

\section{A. Edge Detection}

The edge detection in an input image is essential before the Hough transformation application. As the edge detector, we use Canny and the threshold parameter $T=0.1$ and the Gaussian filter $\sigma=10$. These parameters provide appropriate edge detection in our measurement frames. In Fig. 1 (a) is depicted the measurement frame and its detected edges in Fig. 1 (b).

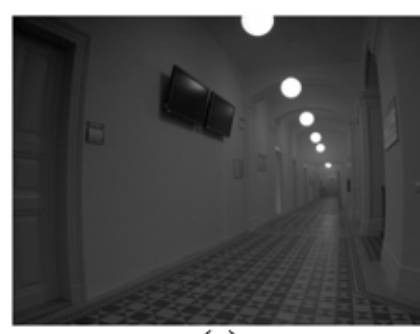

(a)

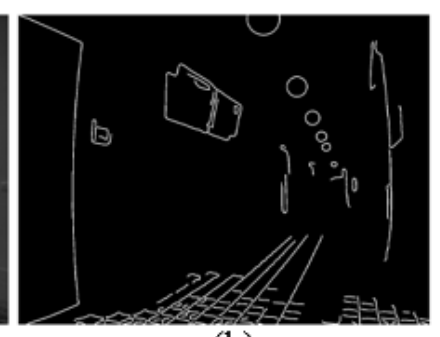

(b)
Fig. 1. Input image, (a) measurement frame, (b) detected edges by Canny.

For the better edges' visualisation, the morphological dilatation by a disk-shaped structuring element with the size 3 is applied. The quality of edges and their amount depends on the input image. Recommendation for its selection are discussed in section with results.

\section{B. Optimal Parametr Estimation}

The algorithm works in iteration steps. In each step the parameter $C P$ is increased by $C P_{S}$ value, set by the user, describing a difference between two $C P$ values. The maximum $C P$ value has to be set before the algorithm start in sufficient range. For example, the user can try undistort an image with $C P=1$ and try to undistort the image with bigger $C P$, where it will be obvious that the strenght is too high.

As it was noted above, focusing only on the maximal local values in the Hough transform accumulator $H_{a}$ during the algorithm's run is not sufficient, because of changing the lines' point count and also two local maxima can be close to each other, their position in $H_{a} x$ axis may change too.

The main idea behind the proposing algorithm give the next Fig. 2 (a) and Fig. 2 (b) comparing the $H_{a}$ for the straight and the curved line.

(a)
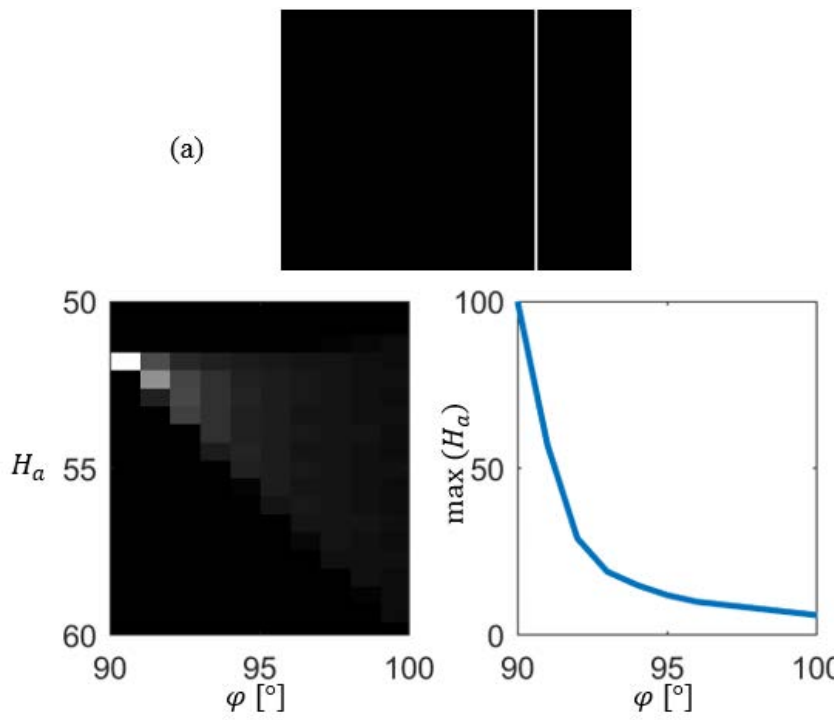

(b)
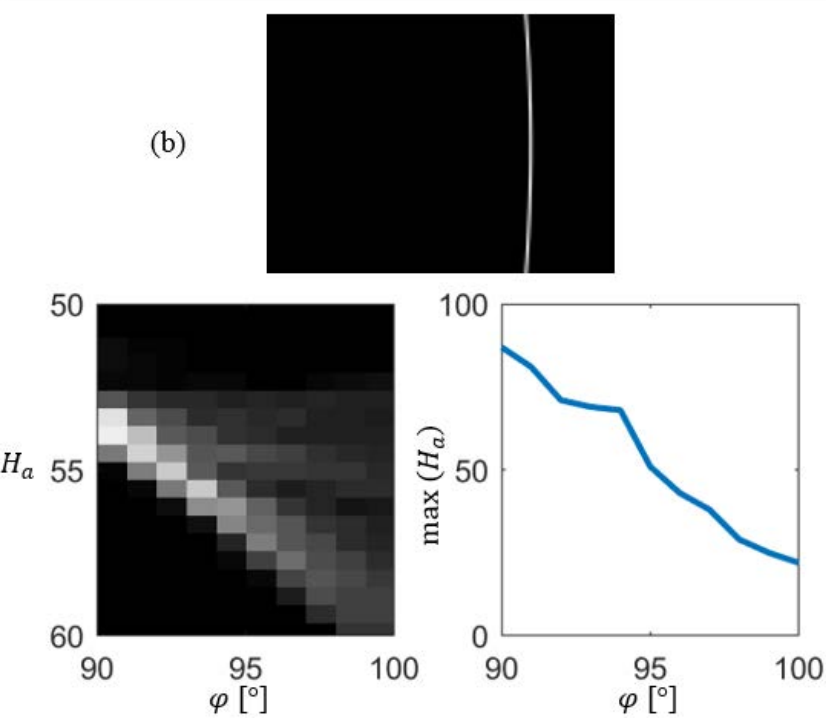

Fig. 2. Main algorithm principle, (a) straight line, (b) curved line, with the coresponding Hough acumulator and its maximum curve.

From comparing of both $H_{a}$ in Fig. 2 is obvious the higher accumulator's maximum value for the straight line, the side values decreasing faster, and their gradient is bigger. The developed algorithm uses this feature for the evaluation of gradients' sum in the defined angle range $A_{r}$. Gradients are estimated from an accumulator's local maximum value. In each iteration step the sum of all gradients is evaluated. The biggest sum value corresponds to the desired optimal $C P$ for 
the actual camera and lens configuration. The accumulator $H_{a}$ is twodimensional, the axis $x$ expressing the selected angle range $A_{r}$ and $y$ axis gives the line's normal vector size expressed as an offset from the accumulator's horizontal centre.

After accumulator's composition in each algorithm's step, it is suitable to suppress its small values lower than threshold $k H_{a_{\max }}$ before its analysis as describing following equation

$$
H_{a}\left(H_{a}<k H_{a_{\max }}\right)=0 .
$$

During the testing the value $k=0.4$ was estimated as the suitable threshold. Values lower than this threshold are equal to zero. For line curvature evaluation the exact position is not important in the $H_{a}$. Even to analyse a gradient in one accumulator's row is meaningless because by curvature influence. One local maximum with close neighbourhood representing a curved line, which normal vectors changing the size reflecting the position changes in the accumulator's both axis, see Fig. 2.

For the analysing purpose the 2D accumulator is transformed into a single vector $\boldsymbol{h}_{\boldsymbol{a}_{\max }}$, where individual values are equal to accumulator's maximum through all columns, thus the elements count is the same as $A_{r}$. The new vector contains several nonzero parts by the application of the equation (1). Next Fig. 3 (a) shows $\boldsymbol{h}_{\boldsymbol{a}_{\max }}$ with $C P=1$ and Fig. 3 (b) with $C P=1.8$ applied on the input image Fig. 1 (a). Mainly the centre part of Fig. 3 (b) has the higher local maximum and all local maxima are narrower in comparison with Fig. 3 (a).
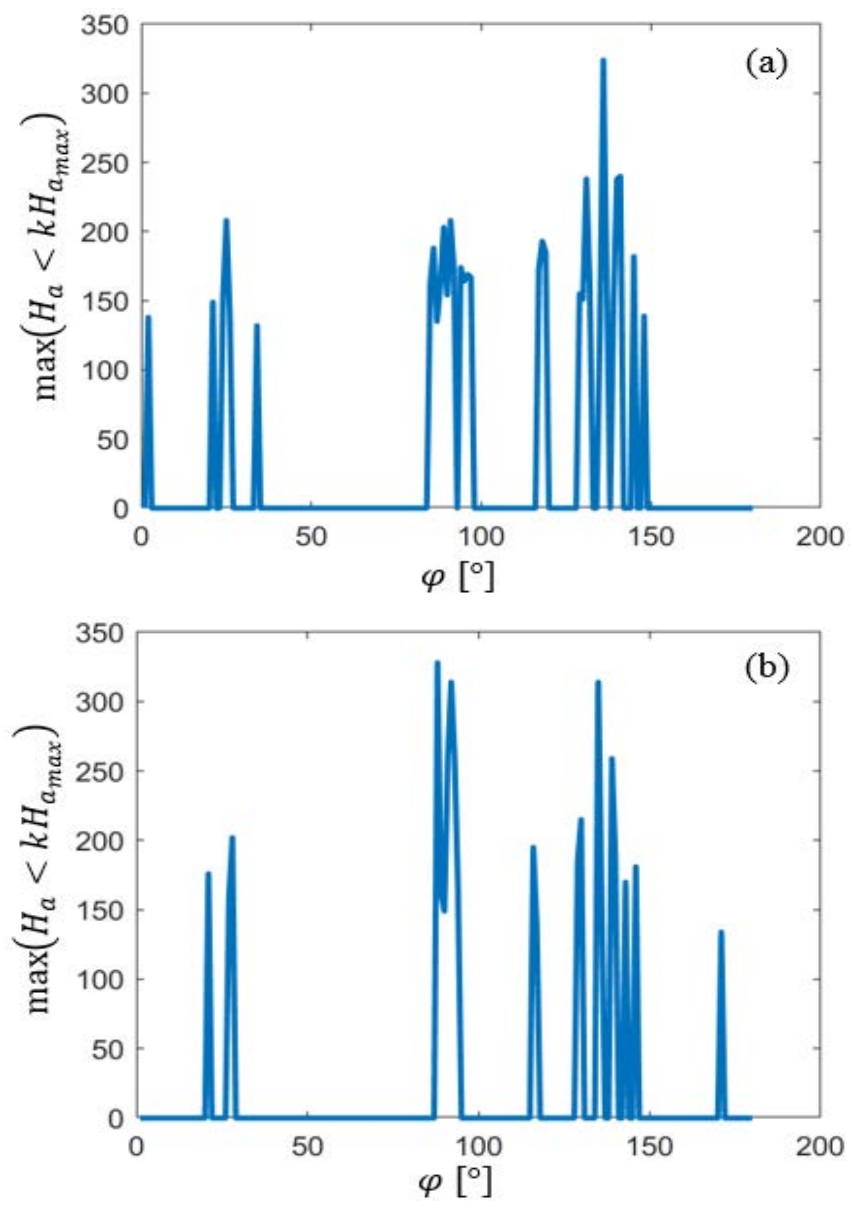

Fig. 3. Comparison $h_{a_{\max }}$ for two $C P$ values, (a) $C P=1$, (b) $C P=1.8$.
For their searching we are using a modified algorithm for the connected component labeling, which accepts close nonzero parts as a one component. The difference between the classical and the modified version is depicted in Fig. 4.

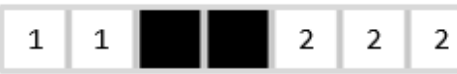

(a)

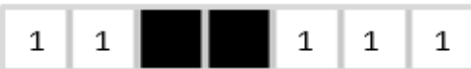

(b)

Fig. 4. Modified connected component labeling algorithm, (a) standard algorithm output, (b) modified output with ignoring zeros.

Parameter $i z$ expressing the maximal count of alowed zero values between two pars to be marked as a one component. In each component, from the set $N$, the maximum value positions are stored in the vector $\boldsymbol{m}_{\boldsymbol{H}_{\boldsymbol{a}}}$. The number of gradients calculated from a one local maxima is defined by parameter $A_{g}$. In other words, the value describes the angle range from a local maximum, where the gradient is calculated. For the optimal distribution of local maxima the $i z=A_{g}$. The following equation (2) describes the gradients' sum $\nabla_{S_{i}}$ calculation for one iteration step

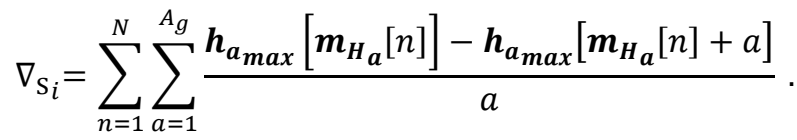

The adding of the index $a$ to a local maximum positions means the gradient evaluation for the maximum's right side. The right side is selected because all lines are searched in the angle range $A_{r}=[0,180]$ and in the accumulator's first left column is $0^{\circ}$.

Fig. 5 shows the curve of gradient $\operatorname{sum} \nabla_{S_{i}}$ for the input Fig. 1 (2). The $C P$ parameter is in the range from 1 to 3 . The best $C P$ value equals to 1.8 .

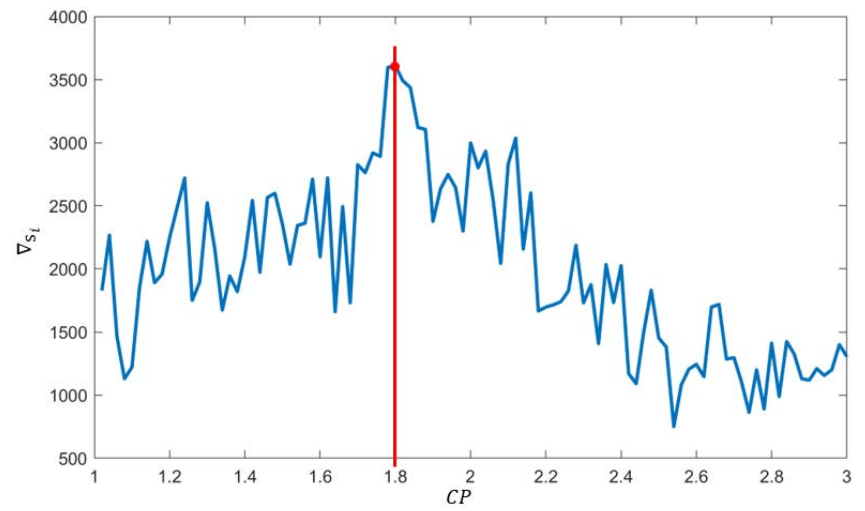

Fig. 5. Algorithm $\nabla_{\mathrm{S}_{i}}$ output for $C P \in[1,3]$.

The described algorithm is able to find the optimal correction parameter even when local maxima change their sizes during the algorithm's iterations. Another testing images and achieved results are evaluated in the next section.

\section{EXPERIMENTAL RESULTS}

Besides the image in Fig. 1 (a), which was used for the algorithm's explanation, the proposed method is tested on the images shown in next Fig. 6. 


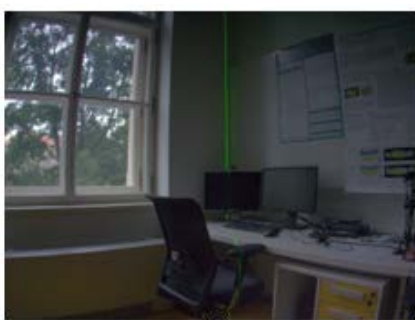

(a)

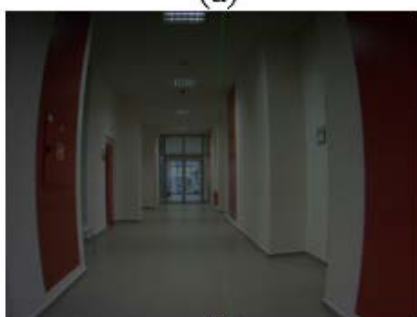

(c)

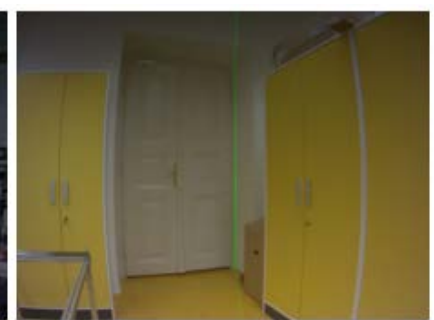

(b)

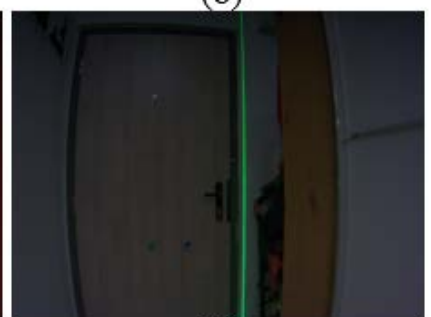

(d)
Fig. 6. Testing images, (a) office, (b) laboratory, (c) coridor, (d) flat door.

In Fig. 7 are shown corresponding images with the edge detection.

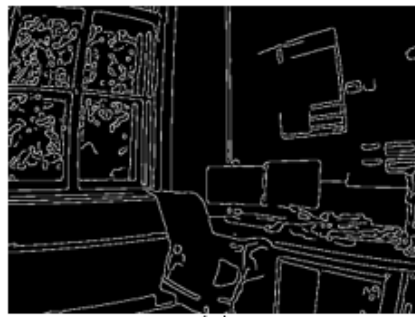

(a)

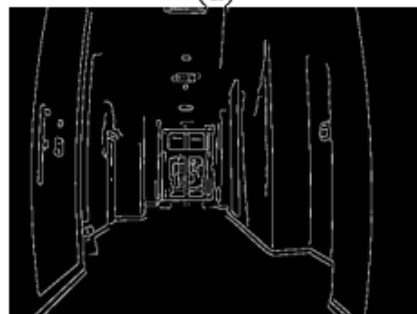

(c)

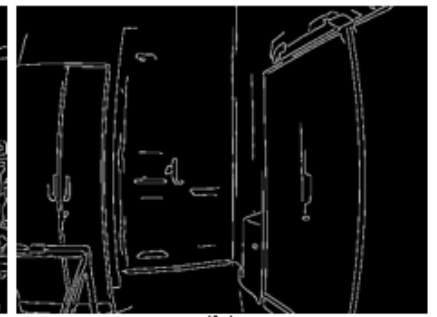

(b)

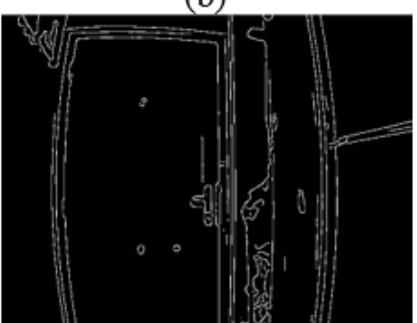

(d)
Fig. 7. Testing images after edge detection, (a) Fig. 6 (a), (b) Fig. 6 (b), (c) Fig. 6 (c), (d) Fig. 6 (d).

Following Fig. 8 shows $\nabla_{S_{i}}$ curves for Fig. 1 and Fig. 6 .

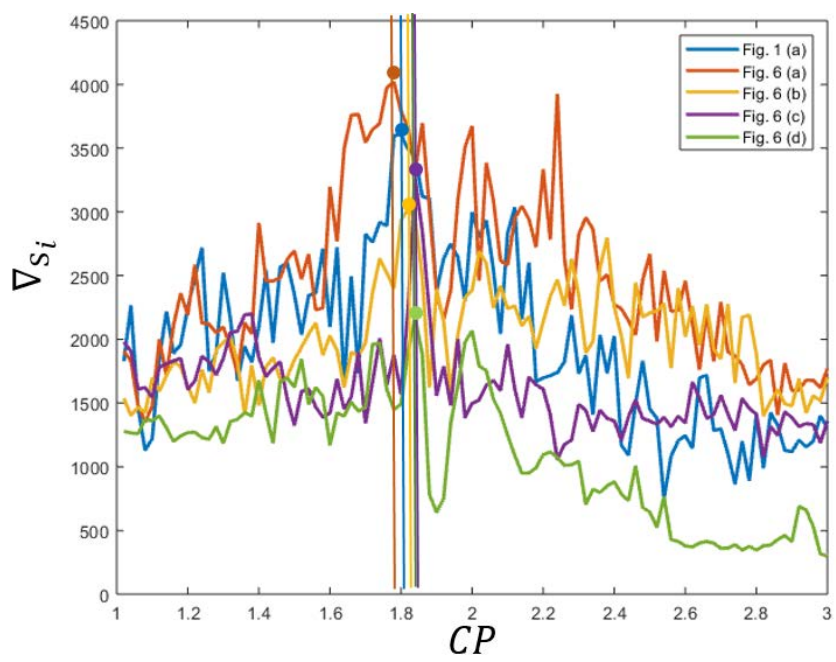

Fig. 8. Resulting $\nabla_{S_{i}}$ curves for testing images from Fig. 1 and Fig. 6.
The testing images in Fig. 1 (a) and Fig. 6 are standard thoroughly selected indoor spaces with presence of edges. Fig. 8 summarize the resultant $\nabla_{S_{i}}$ curves and other important parameters are stated in Tab. 1. For all the testing images the $A_{r} \in[1,3]$ and $C P_{S}=0.02$, which give 100 images for processing.

TABLE I. Estimated PARAMETERS AND PERFORMANCE RESUlts

\begin{tabular}{|c|c|c|c|c|c|}
\hline $\begin{array}{c}\text { Testing } \\
\text { image }\end{array}$ & $\boldsymbol{C P}_{\boldsymbol{E}}$ & $\boldsymbol{m a x}\left(\boldsymbol{\nabla}_{\mathbf{s}_{\boldsymbol{i}}}\right)$ & $\boldsymbol{T}_{\boldsymbol{f}}[\mathbf{m s}]$ & $\boldsymbol{T}_{\boldsymbol{A}_{\boldsymbol{r}}}[\mathbf{s}]$ & $\boldsymbol{\Delta}_{\text {slm }}[\%]$ \\
\hline Fig. 1 (a) & 1.80 & 3608 & 45.6 & 4.62 & 3.82 \\
\hline Fig. 6 (a) & 1.78 & 4019 & 47.9 & 4.83 & 2.52 \\
\hline Fig. 6 (b) & 1.82 & 3022 & 45.1 & 4.56 & 7.57 \\
\hline Fig. 6 (c) & 1.84 & 3295 & 46.5 & 4.69 & 23.3 \\
\hline Fig. 6 (d) & 1.84 & 2171 & 47.7 & 4.78 & 4.81 \\
\hline
\end{tabular}

The first column $C P_{E}$ is the best correction parameter estimation. The second is corresponding $\nabla_{S_{i}}$ value. The next two columns describe the algorithm time consumption, average processing time for one frame $T_{f}$ and for all steps $T_{A_{r}}$ respectively. The last column $\Delta_{\text {slm }}$ expressing the difference between the $\max \left(\nabla_{\mathrm{S}_{i}}\right)$ value and the second local $\nabla_{\mathrm{S}_{i}}$ maximum. This value expressing how the local maxima are close to each other. Because when the sufficient amount of straight lines is not present in the image the algorithm may fail, see Fig. 9 and Fig. 10.

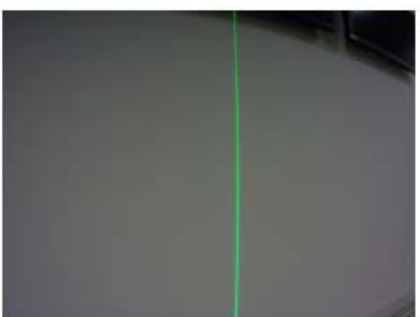

(a)

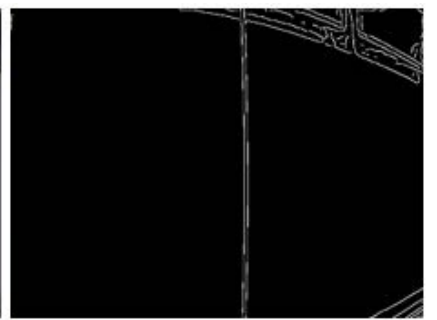

(b)
Fig. 9. Input image with small amount of edges.

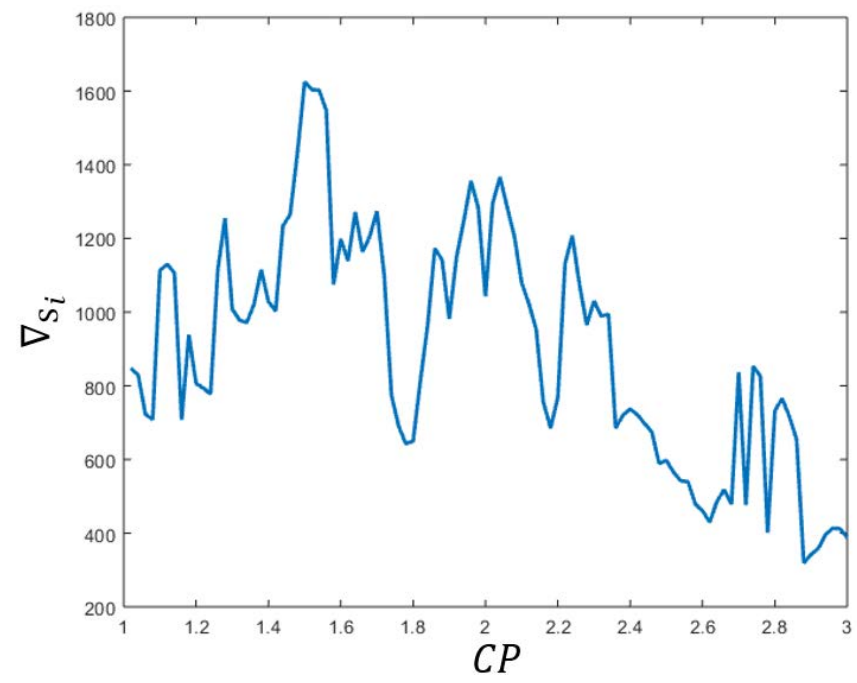

Fig. 10. Resulting $\nabla_{S_{i}}$ curve for Fig. 9.

The Fig. 9 (a) shows an example of the inappropriate input image. Its edge detection is depicted in Fig. 9 (b). The taken image lack of objects with major edges. In comparison the $\nabla_{\mathrm{S}_{i}}$ curve in Fig. 10 with the Fig. 8 is noticeable the different maximum position 1.5 against 1.8 in Fig. 8. Moreover, on the position 1.8 is the local minimum. The main algorithm's assumption for getting the best $\mathrm{CP}$ value estimation is to use 
an image with the sufficient amount of straight lines. When parameters of capturing system are unknown, it is necessary to select the bigger $A_{r}$ range. By the analysis of all $\nabla_{\mathrm{S}_{i}}$ curves in Fig. 8, there is no guarantee, where the position of maximal value in the $\nabla_{S_{i}}$ curve will be, to stop the algorithm. There are several local maxima and minima and it is not possible to predict a curve trend, where the curve will reach the maximum value. This is strongly depending on the input image. We can improve and stabilize the $\nabla_{S_{i}}$ trend by using more suitable scene or by taking an image with a pattern, for example the chessboard as the other researches do. The proposed implementation uses only one image taken from the real scene. The sufficient straight lines' amount is important to ensure the algorithm functionality. When undistorted images from the $A_{r}$ range are available, the algorithm process all images in relatively short time. For the full HD resolution and 100 images, the proposed algorithm finish the processing in the less than $5 \mathrm{~s}$ in the Matlab environment on the CPU Intel Core i5-2410M, 2,3GHz.

\section{CONCLUSION}

In this paper we described the method for the best correction parameter estimation of any optical distortions with known elimination model. The algorithm uses the Hough transform accumulator and the gradient evaluation in its local maxima neighbourhood. No a priori knowledge about the capturing system's parameters is needed. It is only required to take one image with the sufficient amount of straight lines form a real scene. The algorithm is fast because the discrete Hough transform is used. The 2D accumulator's space is transformed into a vector of its maxima through all lines, and gradients are calculated as follows. The proposed method strongly depends on the input image content and may fail if sufficient amount of straight line is not present. The algorithm's evaluation curve is unstable because the local maxima change their positions and sizes. But as we shown on several images, when we use the appropriate image, the algorithm gives stable results.

\section{REFERENCES}

[1] C. Ricolfe-Viala, and A. Sanchez-Salmeron, "Lens distortion models evaluation," Appl. Opt. 49, 5914-5928 (2010).
[2] A. Bismpigiannis, "Measurements and correction of geometric distortion," Stanford University, 2007

[3] X. Dong, Y. Zhang, J. Liu, and G. Hu, "A fisheye image barrel distortion correction method of the straight slope constraint," 2015 8th International Congress on Image and Signal Processing (CISP), Shenyang, 2015, pp. 173-177.

[4] R. Hartley, and S. B. Kang, "Parameter-free radial distortion correction with center of distortion estimation," in IEEE Transactions on Pattern Analysis and Machine Intelligence, vol. 29, no. 8, pp. 1309-1321, Aug. 2007.

[5] C. Choi, H. Lee, and J. Yi, "An interpolation method for strong barrel lens distortion," The Visual Computer, vol. 34, no. 11, pp. 1479-1491, 2018.

[6] J. C. K. Chow, I. Detchev, K. D. Ang, K. Morin, K. Mahadevan, and N. Louie, "Robot vision: calibration of wide-angle lens cameras using collinearity condition and k-nearest neighbour regression," ISPRS International Archives of the Photogrammetry, Remote Sensing and Spatial Information Sciences, vol. XLII-1, pp. 93-99, 2018.

[7] F. Bukhari and M. N. Dailey, "Automatic Radial Distortion Estimation from a Single Image, ” Journal of Mathematical Imaging and Vision, vol. 45, no. 1, pp. 31-45, 2013.

[8] H. Li, and R. Hartley "A non-iterative method for correcting lens distortion from nine-point correspondences," Proc. OmniVision 2005, ICCV-Workshop (2005)

[9] L. Shuhua, and Z. Jun, "GPU-based barrel distortion correction for acceleration," 2013 IEEE 10th International Conference on High Performance Computing and Communications \& 2013 IEEE International Conference on Embedded and Ubiquitous Computing, Zhangjiajie, 2013, pp. 845-848.

[10] P. G. Awade, R. Bodhula, and N. Chopade, "Implementation of barrel distortion correction on DSP in real time," 2016 International Conference on Computing Communication Control and automation (ICCUBEA), Pune, 2016, pp. 1-6.

[11] S. Darvatkar and S. U. Bhandari, "Implementation of barrel distortion correction on FPGA," 2017 International Conference on Computing, Communication, Control and Automation (ICCUBEA), Pune, 2017, pp. 1-6.

[12] A. S. Luthman, D. J. Waterhouse, L. Ansel-Bollepalli, J. Yoon, G. S. D. Gordon, J. Joseph, M. di Pietro, W. Januszewicz, and S. E. Bohndiek, "Bimodal reflectance and fluorescence multispectral endoscopy based on spectrally resolving detector arrays," Journal of Biomedical Optics, vol. 24, no. 03, Mar. 2019.

[13] P. Chmelar, L. Beran, L. Rejfek, and N. Kudriavtseva, "Effective lens distortion correction for 3D range scannig systems," in 2015 57th International Symposium ELMAR (ELMAR), 2015, pp. 37-40. 